\title{
A BRAGG SPECTROMETER FOR STELLAR X-RAY ASTRONOMY*
}

\author{
H. KESTENBAUM, J. R. P. ANGEL**, and R. NOVICK \\ Columbia Astrophysics Laboratory, Columbia University, New York, N.Y. U.S.A.
}

\section{Introduction}

In the last year there have been several proportional counter observations of Sco X-1 which give an indication of an emission feature at around $7 \mathrm{keV}$ (Holt et al., 1969; Acton et al., 1970). The emission lines of $\mathrm{Fe}^{+24}$ and $\mathrm{Fe}^{+25}$, which are very strong in radiation from solar flares of comparable plasma temperature, may be responsible for this feature. Two factors, poor energy resolution and the steeply varying continuum spectrum whose shape is not known in detail, make it impossible to obtain with proportional counters an unambiguous identification of emission features. For this reason we have constructed a Bragg crystal spectrometer to scan the spectrum of Sco X-1. The instrument, flown successfully aboard an Aerobee-170 rocket in April 1970 in conjunction with the Kitt Peak National Observatory, scanned the spectral range from $2.4-2.9 \mathrm{keV}$ which includes the Lyman- $x$ line of hydrogenic sulphur $(2.62 \mathrm{keV})$.The spectrum obtained shows no feature at this energy. The sensitivity of the instrument may be judged from the upper limit $(3 \sigma)$ of 0.08 photon $/ \mathrm{cm}^{2} / \mathrm{s}$ which we are able to place on the line intensity. This value can be compared with the flux of 0.55 photon $/ \mathrm{cm}^{2} / \mathrm{s}$ predicted by Tucker (1967) for the $\mathrm{S}^{+15}$ line intensity from an isothermal, optically thin plasma at $5 \times 10^{7} \mathrm{~K}$. If the line had had this theoretical intensity, we would have observed a $21-\sigma$ signal.

The spectrometer (Figure 1) consists of two large reflecting crystals (each 17 in. $\times 9$ in.) of synthetic graphite mounted on two doors which are deployed when the rocket is above the atmosphere. A scan over a range of Bragg angles is achieved by changing the attitude of the whole rocket, while the doors remain fully opened. Those X-rays which satisfy the Bragg condition are reflected into a central bank of proportional counters, and the detected pulses are telemetered to ground by an FM-FM transmitter. In order to eliminate spurious effects, the rocket makes several scans across the star in different directions. Superposition of scans is accomplished from aspect information from photographs of the star field. To prevent diffuse X-ray background from entering the counters, four aluminum fans open with the doors, and collimators in front of the counters prevent direct illumination of the counters, admitting only those X-rays which are reflected by the crystals.

Since line emission in a hot plasma is a strong function of temperature, it is ne-

* This work was supported in part by the National Aeronautics and Space Administration under Grants NGR 33-008-102, NGR 33-008-012 and Contract NAS 8-24668, and in part by the Air Force Office of Scientific Research under Grant AFOSR-70-1945. It is Columbia Astrophysics Laboratory Contribution No. 34.

** Alfred P. Sloan Research Fellow. 
cessary to know the temperature of Sco X-1 at the time of flight. To determine this temperature, an independent proportional counter experiment is included on the front face of the rocket. Incident X-rays from Sco X-1 are pulse height analysed into 10 energy bins ( 0.6 to $30 \mathrm{keV})$, scaled and telemetered to ground. From these data, best fit temperature for the source is determined, and time variations in intensity are monitored. The results will be presented in another paper.

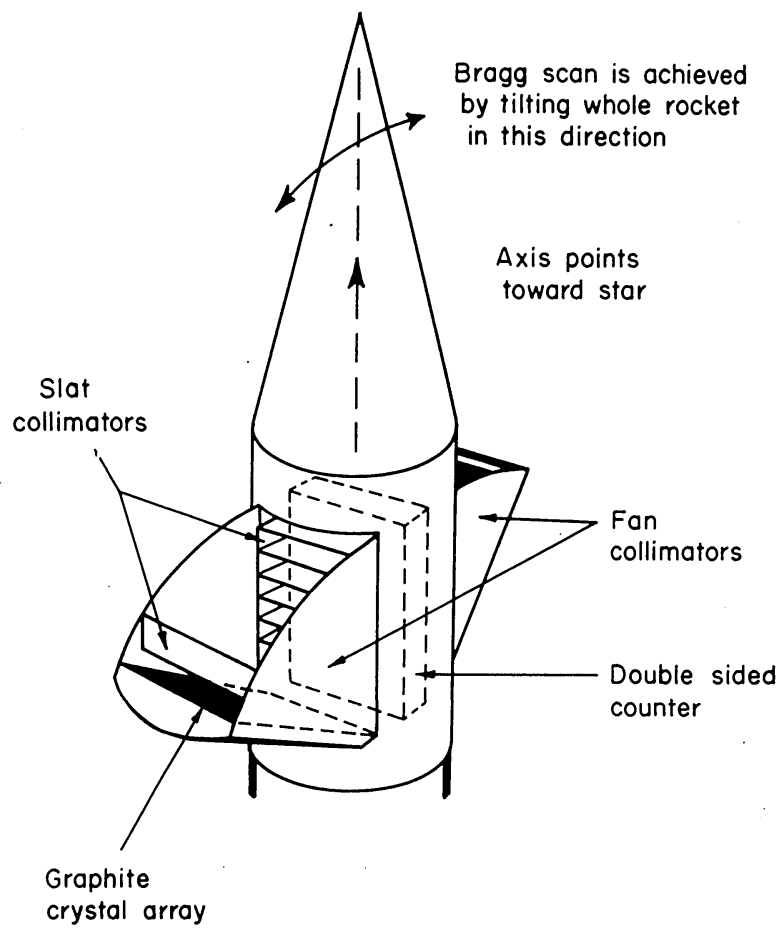

Fig. 1. The spectrometer.

\section{Choice of the Crystal and Estimate of Sensitivity}

Although the $S^{+15}$ Lyman- $\alpha$ line is predicted to be one of the strongest in Sco X-1, a flux of under one photon $/ \mathrm{cm}^{2} / \mathrm{s}$ is all that can be expected, and large detector and crystal areas are needed for a reasonable signal. The instrument has to be designed bearing in mind the constraints of a fixed observing time (about $4 \mathrm{~min}$ ) and poor pointing accuracy. The inertial attitude control system available for this experiment can have pointing errors as large as $\pm 3^{\circ}$, so that a $6^{\circ}$ scan must be made to insure that the desired Bragg angle is covered.

Crystals for Bragg reflection with widely differing properties are available, so one has the opportunity to optimise performance by choosing the most suitable material. In the following section, we derive a simple theoretical formula for the sensitivity of a rocket-borne spectrometer in terms of the crystal and rocket parameters. We consider 
the response of the instrument to line radiation in the presence of stellar continuum radiation, and will show that, under the constraint of a fixed scan angle and time, the peak reflectivity determines the limiting sensitivity independently of the rocking curve width.

In order to calculate the limiting line strength which can be detected, we shall suppose that the observation time is evenly divided over a range, $\phi$, of Bragg angles (in our case $6^{\circ}$ ), and that every detected count is tagged with the Bragg angle at the time of detection. For the purpose of analysis, the scan is divided into equal angular bins of width equal to that of the rocking curve of the crystal (resolution elements). The bins are chosen so that most of the counts detected from the expected line will fall in a single bin centered on the line. An emission line will be detectable if the number of counts $\left(N_{L}\right)$ in the line bin resulting from line photons is significantly larger than the expected statistical fluctuations in the number of counts in the bin from reflected continuum and cosmic ray events. The $3-\sigma$ criterion is given by

$$
N_{L}>3 、 N_{C}+N_{B}
$$

where $N_{B}$ is the number of cosmic ray events and $N_{C}$ is the number of counts from the reflected continuum.

The number of counts in a resolution element at Bragg angle $\theta$ resulting from incident radiation with spectral distribution $I(E)$ is

$$
N=\int_{0}^{x} I(E) R(E, \theta) \varepsilon(E) A(\theta) t(\theta) \mathrm{d} E,
$$

where $I(E)$ is the incident flux at energy $E$ in $\mathrm{keV} / \mathrm{keV} / \mathrm{cm}^{2} / \mathrm{s} ; R(E, \theta)$ is the probability that a photon of energy $E$ incident at angle $\theta$ will be reflected; $\varepsilon(E)$ is the efficiency of the counter at energy $E ; A(\theta)$ is the cross-sectional area of the counter available to detect reflected X-rays at angle $\theta ; t$ is the scan time per resolution element; and $E$ is the photon energy in $\mathrm{keV}$. It can be shown (Angel and Weisskopf, 1970) that for a smoothly varying continuum flux the expression (2) reduces to

$$
N_{c}=I(E) \varepsilon(E) A(\theta) t(\theta) \Delta \theta(E) \cot \theta,
$$

where $E$ is related to $\theta$ by the Bragg condition $E=h c /(2 d \sin \theta),(d$ is the crystal spacing) and $\Delta \theta(E)$ is the integrated reflectivity at incident energy $E$ defined by $\Delta \theta(E)=$ $\int R(E, \theta) \mathrm{d} \theta$.

$\Delta \theta(E)$ can be approximated by $W \times R($ peak), where $W$ is the full width at half maximum of the rocking curve. Writing $t=T W / \phi$, where $T$ is the total scan time and $\phi$ is the total scan expressed in radians, we have

$$
N_{c}=I(E) \varepsilon(E) A(\theta) \cot \theta R(\text { peak }) T W^{2} / \phi .
$$

From Equation (2), the number of counts in the resolution element centered on the 
Bragg angle for a line of strength $I_{L}$ photons $/ \mathrm{cm}^{2} / \mathrm{s}$ is

$$
N_{L} \simeq I_{L} A\left(\theta_{L}\right) \varepsilon\left(E_{L}\right) T R\left(E_{L}, \theta_{L}\right) W / \phi .
$$

From Equation (1), the relevant parameter determining the minimum detectable signal is the ratio

$$
R_{S N}=\frac{N_{L}}{\sqrt{ }\left(N_{C}+N_{B}\right)} .
$$

Because of our background suppression techniques (described later), only $10 \%$ of the cosmic rays within the energy window are accepted as valid events, and we find that the cosmic-ray background rate is less than $20 \%$ of the continuum rate. In the limit where the cosmic-ray background is negligible compared to the continuum

$$
R_{S N} \simeq N_{N_{C}}^{N_{L}} .
$$

Substituting Equations (4) and (5)

$$
R_{S N} \simeq I_{L}\left(\frac{\varepsilon(E) A(\theta) T R\left(E_{L}, \theta_{L}\right)}{I(E) \phi \cot \theta}\right)^{1 / 2}
$$

The rocking curve width $W$ does not appear in this equation; the only crystal property affecting the sensitivity of the instrument to line radiation is the peak reflectivity. For this reason we chose to use graphite crystals, which have a fairly high peak reflectivity combined with a broad $\left(\sim \frac{1}{2}^{\circ}\right)$ rocking curve. The practical advantage of a large rocking curve is that tolerances are not severe, either in the alignment of individual crystal elements which make up a large reflecting panel or in the determination of rocket aspect during the flight. For a $\frac{1}{2}^{\circ}$ crystal, a tolerance of about $\frac{1}{10}^{\circ}$ is adequate.

The shape of the rocking curve of synthetic graphite crystals depends on the degree of misalignment of the individual microscopic domains (Angel and Weisskopf, 1970). Much work has been done in this laboratory and at the Union Carbide Corporation in an effort to alter the crystal mosaic structure by mechanical, thermal, and chemical means. We found that the reflectivity of the best production pieces from Union Carbide could not be improved, and so crystals directly from the production process were used in flight. The characteristic width of the rocking curve at half intensity (FWHM) for an individual one-inch-square crystal fully illuminated in a uniform beam of $\mathrm{Rh} L \alpha$ radiation $(2.7 \mathrm{keV})$, collimated to $0.12^{\circ}$, is about $0.7^{\circ}$, and its peak reflectivity is about $6.5 \%$.

The approximate values for the other parameters in Equation (8) for our measurement are a detector efficiency of 0.7 and area $A$ of $1000 \mathrm{~cm}^{2}$. The scan $\phi$ was over 0.1 radian during a total time $T$ of $4 \mathrm{~min}$. Taking for the continuum at $2.6 \mathrm{keV}$ a flux of $30 \mathrm{keV} / \mathrm{keV} / \mathrm{cm}^{2} / \mathrm{s}$, we obtain a value $I_{L}=0.13 \mathrm{keV} / \mathrm{cm}^{2} / \mathrm{s}\left(0.05\right.$ photon $\left./ \mathrm{cm}^{2} / \mathrm{s}\right)$ for a $3 \sigma$ line intensity. 


\section{The Instrument}

The large graphite crystal panels are constructed from 150 crystals, each one-inch square, and are aligned using optical and X-ray techniques. Each crystal, mounted on its own three-legged stand, is placed on a glass flat in a beam of parallel light, incident at about $45^{\circ}$. The reflected light is focused by a lens onto a screen. The legs of the crystal mount are carefully sanded down until the reflection from the crystal (blurred spot on the screen due to the mosaic property) is centered on the reflection from the glass flat (focused to a point), thus insuring that the crystal face is parallel to the glass flat. The mounted crystal is then fully illuminated in a uniform beam of $\mathrm{Rh} L \alpha$ radiation $(2.7 \mathrm{keV})$, collimated to $0.12^{\circ}$, and the rocking curve is measured. It was found that, for more than $93 \%$ of the first 119 mounted crystals tested, the rocking curve peaks fell within a range of $10 \mathrm{arc}$ min. Optical alignment methods were used exclusively after this correlation had been established. The completed panels were extensively tested, in the CAL 150-ft vacuum facility, using a uniform X-ray beam collimated to 0.12 at two different energies. The measured characteristics are shown in Table I.

\section{TABLE I}

Reflection properties of the spectrometer panels

Panel I

\section{FWHM}

Resolution

Resolving Power

Peak Reflectivity

Size

$\begin{array}{ll}0.65 \cdot 0.05 & 0.75 \mathrm{el} 0.05 \\ 29.9 \mathrm{eV} & 34.5 \mathrm{eV} \\ 87.7 & 75.8 \\ 6.94^{\circ} \circ 0.18^{\circ} \circ & 5.78^{\circ} \circ 0.15^{\circ} \circ \\ 9 \times 17^{\circ} \circ & \end{array}$

Figure 3 shows a Bragg scan with panel $I$ of the $K \alpha$ and $K \beta$ lines produced with a chlorine fluorescence source.

The main bank of proportional counters has 1-mil beryllium windows on both sides and is divided by a grounded wire grid plane to allow the X-rays incident from either side to be distinguished. The counters are filled to 1 atm pressure with a gas mixture chosen to have high opacity at $2.6 \mathrm{keV}$ and good pulse-shape discrimination characteristics. Taking into account the transmission of the windows and the absorption of the gas, the efficiency of the counters is calculated to be $81.5 \%$ at $2.62 \mathrm{keV}$. The total area of beryllium at normal incidence is $1220 \mathrm{~cm}^{2}$. The counters contain a total of 16 anodes which are connected in four groups of four. Each group is assigned its own telemetry channel. The count rates are low enough so that detected pulses can be stretched to a few milliseconds and presented directly to the telemetry, resulting in a maximum dead time of less than $4 \%$. The maximum pulse height is set to correspond to $6 \mathrm{keV}$ photons to insure that second-order reflection at $5.2 \mathrm{keV}$ is 
recorded. The pulse-height information allows one to distinguish first- and secondorder reflection and also to discriminate against background events of the wrong energy.

The anodes on either side of the central ground plane are held in anticoincidence, and risetime discrimination based on pulse-shape differences is used to suppress the cosmic-ray background. The criterion used for risetime acceptance is that the maximum amplitude of the derivative be greater than the maximum amplitude of the attenuated signal. Thus, faster rising pulses (usually due to X-rays) are accepted over slower rising pulses (usually due to cosmic rays). In this risetime rejection scheme, a pulse from the counter is differentiated by means of a clip line, stretched and fed into a comparator where it is compared to a stretched version of the original pulse. If the derivative exceeds the attenuated pulse, the original pulse, with its pulse height still intact, continues to be stretched and fed to the telemetry. Laboratory experiments using different lengths of clip line over the range 20-80 ns showed no noticeable change in acceptance ratios of X-rays to simulated background. Preflight calibrations, using a $\mathrm{Cs}^{137}$ source to simulate the cosmic-ray background, showed that only $10 \%$ of the background events of the right energy are accepted, while $90 \%$ of the true X-ray events are accepted. From flight data obtained before the crystal doors were opened, the residual cosmic-ray background rate was found to be $1.2 \times 10^{-3}$ events $/ \mathrm{keV} / \mathrm{cm}^{2} / \mathrm{s}$ near $2.6 \mathrm{keV}$. Within the energy window chosen for first-order reflection, less than $20 \%$ of the recorded events are due to cosmic-ray background. The pulse-height distribution from one counter while the instrument was pointed at Sco X-1 (Figure 2) shows that the counting rate from both first- and second-order reflection of the continuum is considerably greater than the background rate.

Shadowing of the crystals and counter by the fans, collimators, and rocket body causes the effective area to be reduced for off-axis scan positions. Preflight measurements of these shadowing effects, for various off-axis positions and $6^{\circ}$ scan motions, were made by replacing the proportional counters with large pieces of photo-sensitive paper at the place of the beryllium windows. In an otherwise dark room, a uniform beam of light, collimated to about $\frac{1}{4}^{\circ}$, with cross-sectional area much larger than the crosssectional area of the open door, illuminated the deployed crystal panels. Light was reflected from the crystal panel onto the photosensitive paper just as X-rays would be reflected during flight. By careful measurement of the developed film, the area of the counters illuminated by the reflected beam was measured, and in the reduction of the flight data, these shadowing effects are taken into account.

Aspect information is provided by both a forward-viewing camera mounted on the front plate of the rocket with a clear view of the forward star field and by a wide angle side-viewing camera. Preflight laboratory measurements determine the angular relationships to a precision of $\frac{1}{20}^{\circ}$ between the plane of crystals, the front and side camera mounts, and the rocket axis. The mounted cameras are calibrated with respect to the rocket axis by photographing a known star field and simultaneously sighting two known stars with a transit whose telescope is parallel to the rocket roll axis. To check that the doors open properly, the angle that each door makes with the rocket axis is 


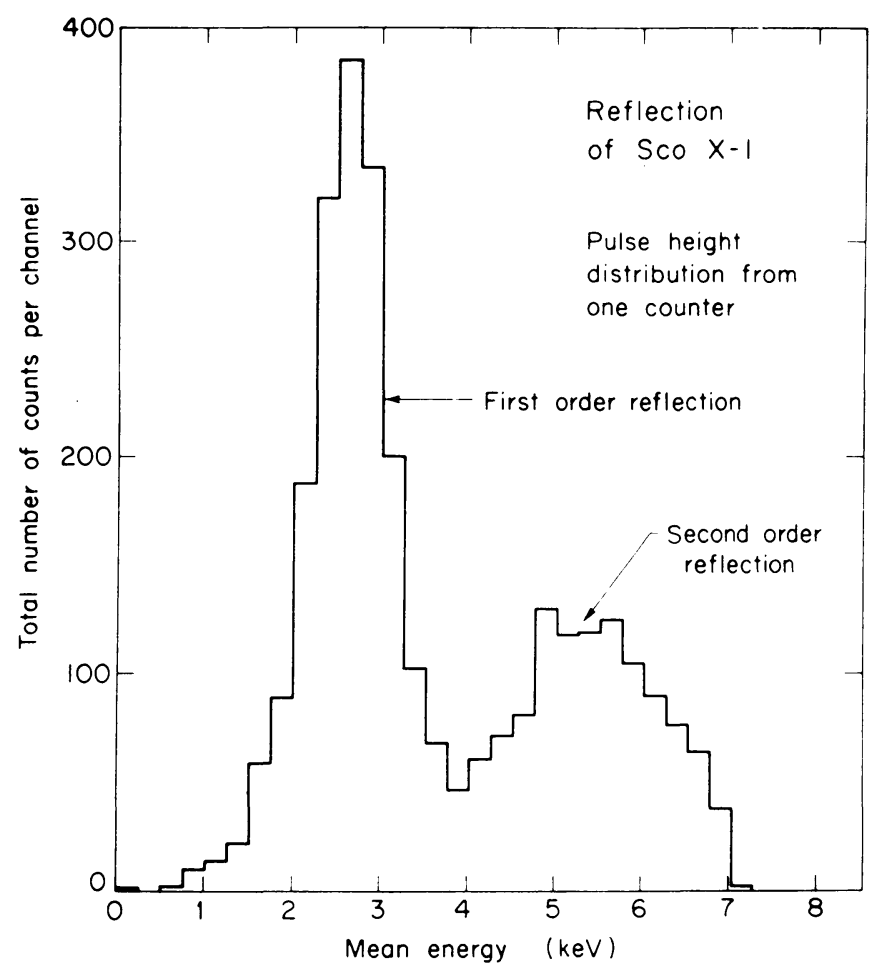

Fig. 2. Pulse-height distribution from one counter during observation of Sco X-1. The two peaks are caused by first- and second-order reflection of the continuum from the crystal. The upper and lower cut-offs in the transmitted data are at 7 and $1.5 \mathrm{keV}$.

monitored by a potentiometer, and a signal is telemetered to the ground with other housekeeping data throughout the entire flight.

Supplementary aspect information is provided by a small, wire modulation collimator and proportional counter mounted on each door. As the rocket scans the sky, this aspect device, fixed securely to the crystal door, modulates the incoming flux sinusoidally with a period of $50 \mathrm{~min}$ of arc. In preflight laboratory measurements using a beam of $\mathrm{Cl} \mathrm{K} \alpha$ and $\mathrm{K} \beta$ radiation, collimated to $0.12^{\circ}$, the response of the crystals and the modulation collimator was measured simultaneously. Thus, a relationship is established between the modulated signal and the Bragg angle for two discrete energies (Figure 3). From the aspect flight data and the photographs, we are then able to determine the exact times that the $S^{+15}$ Lyman- $\alpha$ radiation would be reflected from the crystal. The modulation collimator gives an independent aspect solution tied directly to the crystal aspect which is used to check the camera aspect determinations.

\section{Conclusion}

The results obtained during the flight of the instrument clearly indicate that the Bragg method can yield much higher sensitivity to line emission than can proportional 


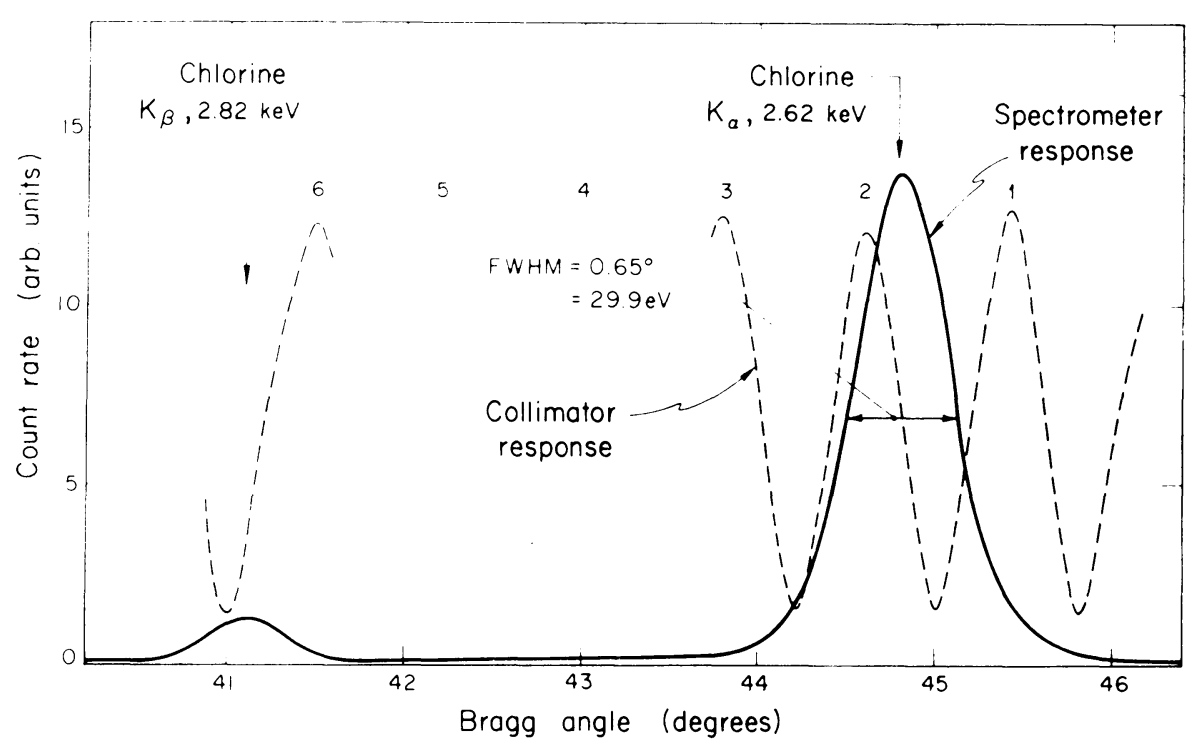

Fig. 3. Laboratory measurement of the reflection from one panel, showing peaks due to the $\mathrm{K}$ i and $\mathrm{K} \beta$ lines of chlorine. The broken line shows the response of the modulation collimator detector permanently fixed to the crystal panel.

counter methods. Our upper limit for the sulphur line emission strength is about $10^{-3}$ of the power in the $2-8 \AA$ continuum, an order of magnitude lower than the best proportional counter limit of Holt et al. (1970) for the iron line emission.

Future work with Bragg spectroscopy should yield a further substantial improvement through the use of very well pointed rockets and more perfect crystals with high reflecting power. Reflectivities of $20 \%$ and more can be achieved in crystals of narrow rocking curve $(\sim 1$ arc min). From Equation (8) it is evident that by reducing the total scan $\phi$ to, say, 6 arc min instead of the $6^{\circ}$ of the present instrument, and by increasing the reflectivity to $20 \%$, an easily realizable value for calcite crystals, an order of magnitude improvement is sensitivity can be achieved.

\section{References}

Acton, L. W., Catura, J. L., Culhane, J. L., and Fisher, P. C.: 1970, Astrophys. J. Letters 161 , L175. Angel, J. R. P. and Weisskopf, M. C.: 1970, Astron. J. 75, 231.

Holt, S. S., Boldt, E. A., and Serlemitsos, P. J.: 1969, Astrophys. J. Letters 158, L155.

Tucker, W. H.: 1967, Astrophys. J. 148, 745. 